HNO $2020 \cdot 68: 838-846$

https://doi.org/10.1007/s00106-020-00917-x Online publiziert: 25. August 2020

(C) Der/die Autor(en) 2020

A. Pudszuhn' $\cdot$ S. Voegeler ${ }^{2} \cdot$ C. Berger ${ }^{2} \cdot$ S. Treskatsch ${ }^{2} \cdot$ S. Angermair ${ }^{2}$ S. Hansen ${ }^{3}$. V. M. Hofmann'

' Klinik für Hals-, Nasen-, Ohrenheilkunde, Campus Benjamin Franklin, Charité Universitätsmedizin Berlin, Berlin, Deutschland

${ }^{2}$ Klinik für Anästhesiologie und Intensivmedizin, Campus Benjamin Franklin, Charité Universitätsmedizin Berlin, Berlin, Deutschland

${ }^{3}$ Institut für Hygiene und Umweltmedizin, Campus Benjamin Franklin, Charité Universitätsmedizin Berlin, Berlin, Deutschland

\title{
Elektive Tracheostomie bei COVID-19-Patienten - Erfahrungen mit einem standardisierten interdisziplinären Vorgehen
}

infektionen besonders leicht, vorrangig durch Tröpfchen $(>5 \mu \mathrm{m})$ möglicherweise auch durch Tröpfchenkerne $(<5 \mu \mathrm{m}$; sog. luftgetragene Übertragung) und Kontaktübertragung [22, 40]. Insbesondere im medizinischen Bereich ist in der Umgebung von COVID-19-Patienten eine Übertragung durch mit respiratorischen Sekreten kontaminierte Oberflächen nicht auszuschließen [25, 40]. Daraus abgeleitet sind die Schutzmaßnahmen für das medizinische Personal: neben der Händehygiene nach WHO-Indikation, die Nutzung einer persönlichen Schutzausrüstung (PSA) bestehend aus einem wasserundurchlässigen Schutzkittel, einem Mund-NasenSchutz, keimarmen Handschuhen und ggf. einer Schutzbrille bei einem Kontakt mit COVID-19-Patienten oder COVID19-Verdachtsfällen ohne eine Aerosolbildung indiziert. Bei allen Behandlungen und Eingriffen, die mit einer Aerosolbildung einhergehen, ist mindestens eine FFP(,filtering face piece“)-Maske und ein Augenschutz in Form einer Schutzbrille oder eines Faceshields zu tragen $[2,28]$. Die konsequente Nutzung dieser Barrieremaßnahmen ist besonders in den Fachrichtungen (z.B. HNO, Anästhesie) angezeigt, in denen aufgrund der Manipulation an den Atemwegen mit möglicher Aerosolbildung ein erhöhtes Expositionsrisiko für das medizinische Personal besteht.

Bereits bei MERS- und SARS-Infektionen und in den sehr stark von der Pandemie mit COVID-19 betroffenen Ländern wurde auf ein besonderes Infektionsrisiko für medizinisches Personal atemwegsnaher Fachrichtungen insbesondere bei Aerosol-induzierenden Maßnahmen hingewiesen $[11,23]$. Daher ist die konsequente Prävention nosokomialer COVID-19-Infektionen von Mitarbeitern im Gesundheitswesen von entscheidender Bedeutung. Gemäß der Mitteilung des italienischen Chirurgenverbandes FNOMCEO (Federazione Nazionale degli Ordini dei Medici Chirurghi e degli Odontoiatri) sind bis zum 22. April 2020 infolge der COVID-19Pandemie 151 Ärzte in Italien gestorben [39]. Bei einer zu diesem Zeitpunkt dokumentierten Gesamtzahl von 26.977 Todesfällen durch COVID-19 entspricht das einem Anteil an Ärzten von 0,56\% (weiteres medizinisches Personal ausgenommen). Ende März 2020 lag der Gipfel der Infektionsrate beispielsweise in Italien in den Gesundheitsberufen bei etwa $10 \%$ mit deutlichen regionalen Unterschieden, wobei die Infektionsquelle 
möglicherweise nicht nur von Patienten ableitbar ist [5].

Die meist notwendige invasive Langzeitbeatmung schwerstkranker COVID19-Patienten kann im Verlauf die elektive Anlage einer Tracheostomie (TS) erfordern [14]. Dieser Eingriff birgt durch die Möglichkeit der virushaltigen Aerosolbildung ein besonders hohes Risiko der SARS-CoV-2-Exposition für das medizinische Personal [14, 28, 38]. Aufgrund der individuell unterschiedlichen Erkrankungsverläufe ist der Zeitpunkt einer TS nicht immer in einer Phase der geringeren COVID19-Erkrankungsaktivität oder erst nach erfolgtem Ausschluss fortbestehender Viruslast planbar. Deshalb kommt neben den genannten Schutzmaßnahmen der Risikominimierung bzw. kontrollierten Freisetzung von potenziell infektiösem Aerosol eine entscheidende Rolle zu [21, 36].

Orientierend an den bekannten Daten der SARS-Epidemie haben mehrere nationale HNO-Gesellschaften bereits Empfehlungen zur TS bei COVID-19 ausgesprochen $[13,14,16,18,21,30,32$, 33]. Die publizierten Guidelines und Protokolle zeigen jedoch auch unterschiedliche Standards [4, 10, 14, 17, 24, 35]. Zur Risikominderung im Rahmen der COVID-19-Pandemie ist es zusätzlich zu diesen Empfehlungen wichtig, eine lokale, gut strukturierte und an die Ressourcen der Klinik angepasste Verfahrensweise $\mathrm{zu}$ erstellen.

\section{Standardisiertes Vorgehen}

Durch ein standardisiertes Vorgehen bei einer elektiven TS von COVID-19-Patienten im Sinne einer Standard Operating Procedure (SOP), wurde ein Ablauf festgelegt, der insbesondere im Bereich kritischer Schritte mit potenziellen Auswirkungen auf die Gesundheit des medizinischen Personals präventive Maßnahmen bestimmt. Diese SOP legt detailliert die prozedurspezifischen Erfordernisse unter Berücksichtigung der klinikinternen Gegebenheiten fest und wird durch die beteiligten Disziplinen konsentiert. Außerdem muss diese SOP bedarfsabhängig einer regelmäßigen Prüfung unterliegen. Im Fall der SOP zur TS bei COVID-19-
Patienten sind in diesen Prozess die operativen Fachrichtungen, die Anästhesiologie/Intensivmedizin, das operative als auch anästhesiologische Pflegepersonal und die Krankenhaushygiene einbezogen.

Klinikintern wurde die offene TS in tiefer Narkose und Vollrelaxation als Methode der Wahl favorisiert, da sie sowohl den Vorteil einer verkürzten und kontrollierten Aerosolexpositionszeit und -menge als auch die Platzierung großlumiger Trachealkanülen (TK) bietet. Im Fall von SARS-CoV-2-positiven Patienten ist sie der dilatativen Tracheotomie aufgrund der Möglichkeit zur chirurgischen Blutstillung (Patienten mit Antikoagulation), längeren Apnoezeiten, häufigeren Diskonnektionen der Beatmung und dauerhaft erforderlicher bronchoskopischer Kontrolle vorzuziehen [8, 37].

Des Weiteren wurde klinikintern festgelegt, dass eine TS bei COVID-19-Patienten nur im Operationssaal durchgeführt wird, um die Vorteile der dortigen Raumlufttechnik zur Abführung der z. B. durch Aerosole belasteten Luft, der optimalen Lagerung des Patienten und der uneingeschränkten Positionierung des Operationsequipment zu nutzen [20]. Die zuführenden Operationssaaltüren werden für den Zeitraum der Operation allseitig mit dem Warnhinweis auf die Behandlung eines COVID-19-Patienten beschildert und bleiben durchgehend während der Operation geschlossen. Nicht benötigtes Equipment wird aus dem Operationssaal entfernt. Grundsätzlich sind TS auf Intensivstation (ITS) auch möglich, jedoch ist derzeit ungeklärt, ob sie Vorteile bezüglich eines geringeren Infektionsrisikos bringen [14].

Zusätzlich zur empfohlenen sterilen flüssigkeitsundurchlässigen langärmligen Operationsbereich-Schutzkleidung und sterilen Operationshandschuhen tragen alle am Eingriff Beteiligten entsprechend der WHO-Empfehlungen Gesichtsschutz (immer Faceshield) und mindestens eine FFP2- oder -3-Maske ohne Ausatemventil als PSA [2, 20]. Die Effektivität der Infektionsschutzes ist entscheidend vom Test eines korrekten Maskensitzes („fitting test“) abhängig [14]. Ist dies anatomisch nicht möglich, sollte die TS durch einen anderen Opera- teur erfolgen. Eine gegenseitige Kontrolle der korrekten Anlage aber auch der postprozeduralen Ablage der PSA inklusive der Händedesinfektion nach Berühren des Kittels und der Maske sowie vor möglichen Gesichtskontakten im Sinne eines „buddy check“ wird empfohlen. Es ist empfehlenswert, die konkreten Abläufe durch eine externe Supervision der Krankenhaushygiene zu prüfen, um mögliche Lücken in der Umsetzung identifizieren zu können [15].

Um ein sicheres operatives und anästhesiologisches Management zu ermöglichen und das Risiko einer Infektion des medizinischen Personals zu minimieren, werden nur erfahrene Anästhesisten und HNO-Operateure sowie im Umgang mit der SOP geschultes Personal eingesetzt. Zum Team gehören immer 6 Personen (Anästhesist, Anästhesiepflege, Operateur, Operationsassistenz, Operationspflege steril und unsteril).

Zur Durchführung einer elektiven TS bei SARS-CoV-2-positiven Patienten ist eine interdisziplinäre Planungsphase erforderlich, welche prä-, intra- und postoperative Erfordernisse erfasst.

\section{Präoperative Phase}

Der Zeitraum dieser Phase kann mehrere Tage umfassen. Bei der Indikationsstellung einer TS unter COVID-19 spielt der Aktivitätsstatus der Erkrankung eine wesentliche Rolle, um das Risiko einer nosokomialen Infektion der Beteiligten zu reduzieren [14]. Aus anästhesiologischer Sicht ist insbesondere die Einschätzung der Apnoetoleranz zur hinreichenden Oxygenierung neben der grundsätzlichen klinischen Stabilität des Patienten für die Narkose entscheidend. Die erforderlichen anästhesiologischen und operativen Maßnahmen einschließlich Apnoephasen müssen sicher toleriert werden. Zudem können verschiedene Parameter (schwierige Re-Intubation bei Tubusdislokation, Druckläsionen durch den Tubus in Bauchlage, CuffDichtigkeitsprobleme) die Indikation zur TS beeinflussen. Es sollte ein möglichst günstiger Zeitpunkt der bereits in Deeskalation befindlichen Beatmungstherapie vorliegen. Der HNO-Operateur muss präoperativ insbesondere Neben- 
erkrankungen (Gerinnungsstörungen durch Hämodialyse, Antikoagulation, Thrombozytopenie, Anämie) und die anatomischen Besonderheiten beachten (Adipositas, Schilddrüsenoperationen, Reklinationsmöglichkeiten, Bildgebung), um intraoperativ ein sicheres und rasches Vorgehen zu ermöglichen. Da thrombembolische Ereignisse bei COVID-19 erhöht sind, wird eine moderate Antikoagulation empfohlen [19]. Sowohl durch Sepsis und Hämodialyse als auch extrakorporale Membranoxygenierung (ECMO) sind Gerinnungsstörungen bei COVID-19-Erkrankten aggraviert [14]. Die Koagulopathien, wie Thrombozytopenie, Verlängerung der Blutungszeit durch Antikoagulation, sollten für den Operationszeitpunkt optimiert werden, um intraoperative Blutungskomplikationen $\mathrm{zu}$ reduzieren. Auf Grundlage dieser Informationen werden Indikation und Zeitpunkt des Eingriffs gemeinsam interdisziplinär festgelegt.

Für alle Beteiligten muss ausreichend PSA vorhanden sein. Alle erforderlichen Operationsmaterialen (Tracheostomiesieb, TK einschließlich Ersatz in verschiedenen Größen, virendichte Absaugfilter für Rauchabsaugung, PSA, sterile Abdeckung) werden in einem Container dauerhaft deponiert und sind so auch in Notfällen griffbereit. Zur Konnektion an die TK müssen die erforderlichen Anteile des Beatmungssystems („head and moisture exchange filter", HME-Filter, geschlossenes Absaugsystem, Winkelstück und Tubusverlängerung) sowie ein individuell festgelegter Endotrachealtubus (ET) für den Fall einer CuffBeschädigung vorhanden sein (• Abb. 1).

Es werden entsprechend intensivmedizinischer Standards blockbare, ungefensterte TK mit subglottischer Absaugung gewählt, um eine dauerhafte peristomale Sekretreduktion während der fortgesetzten Beatmungstherapie zu erreichen [1].

\section{Intraoperative Phase}

Zum maßvollen Umgang mit Zeit- und Material(PSA)-Ressourcen wird der Patient von der ITS direkt durch das erfahrene und speziell für COVID-19-Patienten geschulte anästhesiologische CO-

HNO 2020 - 68:838-846 https://doi.org/10.1007/s00106-020-00917-x

(c) Der/die Autor(en) 2020

A. Pudszuhn · S. Voegeler · C. Berger · S. Treskatsch · S. Angermair · S. Hansen · V. M. Hofmann

Elektive Tracheostomie bei COVID-19-Patienten - Erfahrungen mit einem standardisierten interdisziplinären Vorgehen

\section{Zusammenfassung}

Berichtet wird über die Erfahrungen mit einer interdisziplinären klinikinternen SOP (Standard Operation Procedure) zur Tracheostomie (TS) bei "Coronavirus-Disease"(COVID19)-Patienten, unter Berücksichtigung der allgemeinen nationalen und internationalen Empfehlungen. Der interdisziplinär festgelegte operative Zeitpunkt der TS aufgrund einer prolongierten invasiven Beatmung und frustranen Weaning-Versuchen betraf Phasen sowohl hoher als auch niedriger Erkrankungsaktivität. Es wurden 5 TS bei Patienten mit einem Durchschnittsalter von 70,6 Jahren durchgeführt. Neben den
Standard-COVID-19-Schutzmaßnahmen für das medizinische Personal zur Vermeidung einer nosokomialen COVID-19-Infektion führt die SOP-unterstützte Kommunikation während der TS zu einer periprozeduralen Sicherheit aller Beteiligten. COVID-19Erkrankungen des medizinischen Personals der beteiligten Abteilungen sind bisher nicht bekannt.

Schlüsselwörter

Tracheotomie · SARS-CoV-2 $\cdot$ Medizinisches Personal $\cdot$ SOP $\cdot$ Coronavirus

\section{Elective tracheostomy in COVID-19 patients: experience with a standardized interdisciplinary approach}

\section{Abstract}

Experience with an interdisciplinary SOP (standard operating procedure) for tracheostomy (TS) in COVID-19 patients, taking into account the general national and international recommendations, is reported. The operative timing of TS due to prolonged invasive ventilation and frustrating weaning attempts was determined on an interdisciplinary level and involved phases of both high and low disease activity. Five TS were performed in patients with an average age of 70.6 years. In addition to the standard COVID-19 protective measures for medical staff to avoid nosocomial COVID-19 infection, SOP-supported communication during the TS leads to periprocedural safety for all involved. COVID-19 infections among medical staff in the departments involved are not yet known.

\section{Keywords}

Tracheotomy - SARS-CoV-2 $\cdot$ Healthcare staff . SOP. Coronavirus
VID-Airway-Team in den Operationssaal transferiert.

Nach sorgfältiger Umbettung und Lagerung des Patienten erfolgt gemäß WHO-Checkliste das Team-Time-Out, in dem neben den speziellen COVID19-Infektion weitere patientenbezogene Besonderheiten Erwähnung finden. Intraoperativ regelt ein klarer Workflow die Abläufe. Der gesamte Eingriff wird in ausreichend tiefer Sedierung und Relaxierung unter Neuro- und Relaxationsmonitoring („train of four“: TOF =0) durch den Anästhesisten durchgeführt. Vor jeder potenziell aerosolfreisetzenden Maßnahme erfolgt eine unmissverständliche Kommunikation über den Operationsfortschritt und relevante anästhesiologische Maßnahmen in Zusam- menhang mit der Beatmung (• Abb. 2). Es ist zu beachten, dass die Verständigung aufgrund der FFP-Masken und dem Faceshield erschwert sein kann. Die Operationsschritte, wie das Vorschieben des ET, die Inzision der Trachea sowie das Einführen der TK, müssen immer nach standardisierter Vorbereitung des Patienten und höchster Aufmerksamkeit aller Beteiligten in Apnoe erfolgen. Dabei ist insbesondere die interdisziplinäre Interaktion bei der Inzision der Trachea entscheidend. Eine Cuff-Beschädigung ist zwingend $\mathrm{zu}$ vermeiden. Ohne abgedichtete Trachea sollte keine Beatmung erfolgen, um eine Gefährdung des Personals durch Exposition virushaltiger Aerosolbildung zu vermeiden. Ein verlängertes Aussetzen der Beatmung 


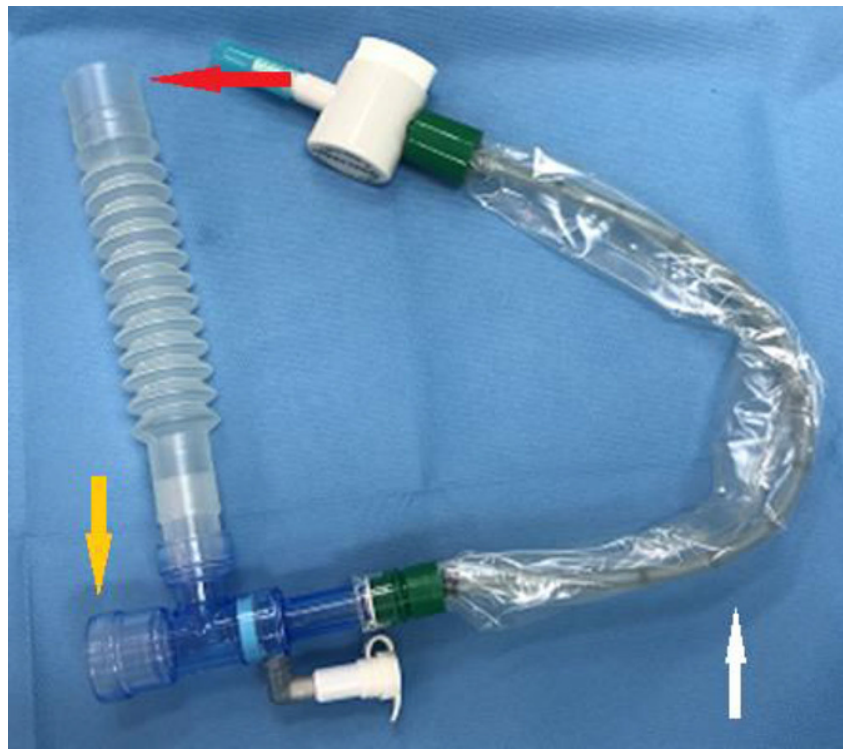

Abb. $1<$ Geschlossene Absaugung (Pfeil weiß), Anschluss an Trachealkanüle (Pfeil gelb), Anschluss zum $\operatorname{HME}($,head and moisture exchange filter")-Filter (Pfeil rot)

hingegen resultiert in Oxygenierungsproblemen und gefährdet damit die $\mathrm{Pa}$ tientensicherheit. Zur Vermeidung einer Cuff-Beschädigung sind exakte Schritte in der SOP formuliert (• Abb. 2). Sollte der Cuff jedoch dennoch beschädigt werden, ist eine sofortige transstomale Sicherung des Atemwegs mit dem vorab bereitliegenden ET durch den Operateur möglich, um eine für das Personal gefahrlose und den Patienten angepasste Beatmung zu beginnen. Wenn operationstechnisch möglich, wird die TS bei liegendem transstomalen ET beendet. Alternativ kann durch den Anästhesisten unter Fortsetzung der Beatmung und stabilen Verhältnissen z.B. über einen Wechselstab ein neuer transglottischer ET platziert und die TS kann beendet werden.

Die Blutstillung sollte möglichst durch Unterbindungen erfolgen. Bei Kauterisierung wird eine permanente Absaugung mit einem speziellen virendichten Rauchpartikelfiltersystem durchgeführt (Richtlinien und klinische Untersuchungen zu COVID-19 ausstehend). Die tracheokutane Anastomose wird mit resorbierbarem Nahtmaterial (Polydioxanone) verschlossen. Die Trachealkanülenfixierung erfolgt zusätzlich per nichresorbierbarer Naht, um eine Dislokation bei Beatmungen in Bauchlage $\mathrm{zu}$ vermeiden.

In Apnoe erfolgt nach Einführung und Blockung der TK die Konnektion

\section{Erfahrungen mit Tracheostomien von COVID-19-Patienten}

Seit Einführung der SOP wurden 5 Patienten (1 weiblich, 4 männlich) mit COVID-19-Erkrankung tracheostomiert (• Tab. 1). In allen Fällen erfolgten präoperativ eine interdisziplinäre Einschätzung der Indikation und die Festlegung des optimalen Operationszeitpunktes aus anästhesiologischer und operativer Sicht. Das Durchschnittsalter der Patienten beträgt 70,6 Jahre (Spannweite 57-77 Jahre). Bei allen 5 Patienten war zum Operationszeitpunkt noch SARS-CoV-2-RNA in der PCR aus dem tiefen (bronchoalveoläre Lavage/Tracheobronchialsekret) und oberen Respirationstrakt (Nasen-RachenAbstrich) nachweisbar. Alle Patienten hatten eine Anämie und eine Koagulopathie, 2 Patienten eine Thrombozytopenie. Der durchschnittliche Body-MassIndex (BMI) betrug $26,3 \mathrm{~kg} / \mathrm{m}^{2}, 3$ Patienten wiesen eine Präadipositas auf, ein Patient eine Adipositas Grad I. Alle Patienten hatten mindestens eine pulmonale bzw. kardiovaskuläre Vorerkrankung. In 3 Fällen waren anamnestisch operative Eingriffe am Tag (Fall 2: Iliacalaneurysmaresektion) bzw. in den Wochen (Fall 4/5: popliteocruraler Venenbypass, Perkutane transluminale Koronarangioplastie mit Stenteinlage) vor der stationären Aufnahme als COVID-19Patienten vorausgegangen.

Die Erkrankungsverläufe der Patienten zeigen eine mittlere Zeitspanne vom Beginn der Symptome bis zur erforderlichen intensivmedizinischen Überwachung von durchschnittlich $3 \mathrm{Ta}$ gen (Spannweite 1-7 Tage, Ausschluss Patient 4, anamnestisch aufgrund deliranter Symptomatik bei ITS-Aufnahme $\mathrm{zu}$ erheben; - Abb. 3). Aufgrund der zunehmenden respiratorischen Insuffizienz dauerte es nach Aufnahme auf der ITS bis zur Intubation durchschnittlich 2,8 Tage (Spannweite 2-4 Tage). Der Zeitraum von der Intubation bis zur operativen TS betrug durchschnittlich 13 Tage (Spannweite 7-21 Tage).

Im Fall 1 einer 57-jährigen Patientin wurde die Indikation zur frühen TS in dem Zeitintervall einer verhältnismä- 


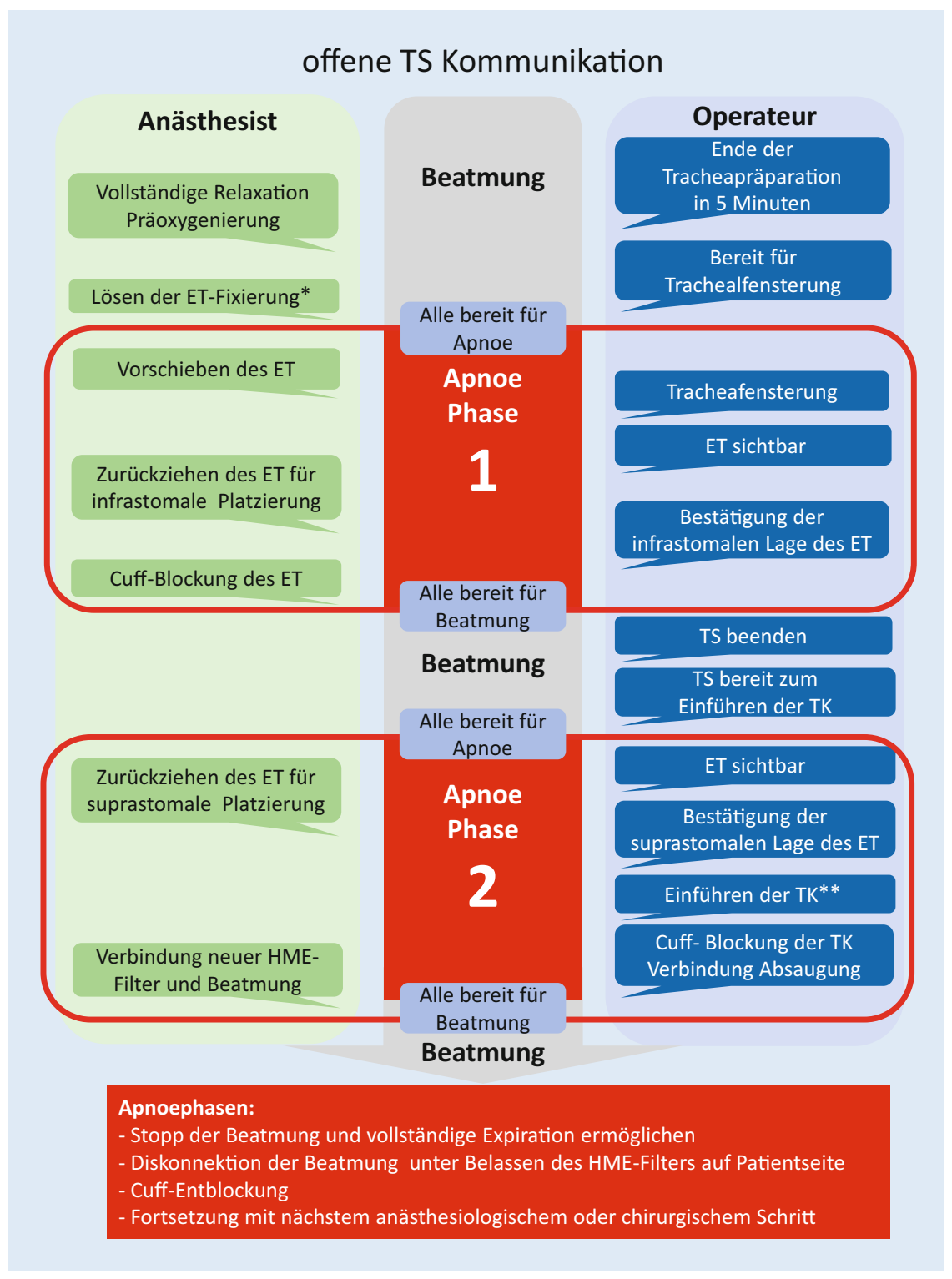

Abb. 2 A Intraoperativer Kommunikationsfluss nach SOPTracheostomie(TS) bei COVID-19-Patienten. ETEndotrachealtubus, TK Trachealkanüle. * ab hier, permanente manuelle ET Sicherung durch das Anästhesieteam; ${ }^{* *}$ ab hier, permanente manuelle TK Sicherung durch den Operateur bis zur endgültigen Fixierung

Big hohen Erkrankungsaktivität gestellt. Bei der Patientin mit einem BMI von $>30 \mathrm{~kg} / \mathrm{m}^{2}$ war eine prolongierte Weaningphase zu erwarten (• Abb.4). Durch den ET bei Beatmung in Bauchlage zeigten sich trotz präventiver Maßnahmen sehr frühzeitig Druckulzera der Mundwinkel beidseits und zudem Schwellungen der Zunge. In dieser Situation waren aufgrund der Adipositas und der Zungenschwellung weder eine extrathorakale noch transösophageale Echokardiographie zur Beurteilung der Herzfunktion on durch Umlagerungen so gering wie möglich zu halten, wurde abweichend von der SOP interdisziplinär entschieden, die operative TS am Patientenbett auf ITS durchzuführen. Präoperativ erhielt der Patient zur Gerinnungsoptimierung Thrombozytenkonzentrate. Dieser ECMO versorgte Patient verstarb 14 Tage nach der TS infolge einer Candidasepsis und multiplen anderen Organschäden.

Zwei Patienten konnten nach erfolgreichem Weaning 1 (Fall 4) bzw. 3 (Fall 1) Woche/n nach TS in eine weiterbehandelnde Klinik bzw. Frührehabilitation verlegt werden. Trotz erfolgreichem Weaning nach TS ist bei 2 Patienten (Fall 2 und 5) 3 bzw. 4 Wochen postoperativ eine weitere intensivmedizinische Behandlung erforderlich (nicht SARS$\mathrm{CoV}-2$ verursachte Erkrankungen, z. B. Zosterenzephalitis, akutes Nierenversagen). Tendenzen bezüglich verbesserter Weaningverläufe der Patienten nach TS sind aufgrund der geringen Fallzahl und der besonderen Fallkomorbiditäten nicht ableitbar.

Bei 4/5 TS war sowohl der intra- als auch postoperative Verlauf komplikationslos. Im Fall 2 musste 14 Tage postinterventionell eine Blutung infolge einer lokalen Wundheilungstörung operativ versorgt werden.

Es wurden 4/5 TS und die TS-Nachblutung von einem Operateur (eine OP durch anderen Operateur) und jeweils 3 verschiedenen Operationsassistenzen durchgeführt. In allen Fällen wurde ein erfahrenes und SOP-geschultes, aber personell nicht konstantes Anästhesieteam sowie anästhesiologisches und Operationspflegepersonal eingesetzt. Dies entspricht auch dem klinischen Alltag (Personal in Schichtsystemen), in dem die Versorgung sowohl geplanter als auch Notfalleingriffe an den Atemwegen jederzeit bei COVID-19-Patienten gewährleistet werden muss. Die konsequente Anwendung der SOP vermittelte allen Beteiligten subjektiv Sicherheit im Umgang mit der infektiologisch besonderen Situation. Aufgrund der im Fall 3 intraoperativ kurzzeitig aufgetretenen Beatmungsleckage bei Manipulation an der Trachea ohne Hinweis auf eine Cuff-Beschädigung, erfolgte 5 Tage postoperativ eine vorsorgliche Nasen- 


\begin{tabular}{|c|c|c|c|c|c|c|c|}
\hline Patient & $\begin{array}{l}\text { Alter (J) } \\
\text { m/w }\end{array}$ & $\begin{array}{l}\text { Indikation zur Tracheo- } \\
\text { stomie }\end{array}$ & Nebendiagnosen & $\begin{array}{l}\mathrm{BMI} \\
\mathrm{kg} / \mathrm{m}^{2}\end{array}$ & $\begin{array}{l}\mathrm{Hb} \\
\mathrm{g} / \mathrm{dl}\end{array}$ & $\begin{array}{l}\text { Thrombo- } \\
\text { zyten/nl }\end{array}$ & $\begin{array}{l}\text { PTT } \\
\text { s }\end{array}$ \\
\hline 1 & $\begin{array}{l}57 \\
W\end{array}$ & $\begin{array}{l}\text { Hohe Beatmungsdrücke, } \\
\text { ausgeprägte Druckläsionen } \\
\text { Mundwinkel und Zungen- } \\
\text { schwellung durch ET }\end{array}$ & $\begin{array}{l}\text { Asthma bronchiale } \\
\text { Bipolare Störung } \\
\text { Z.n. Schilddrüsenresektion }\end{array}$ & 30,8 & 8,1 & 96 & 40,2 \\
\hline 2 & $\begin{array}{l}76 \\
M\end{array}$ & $\begin{array}{l}\text { Prolongierte ET-Intubation } \\
\text { Frustranes Weaning }\end{array}$ & $\begin{array}{l}\text { Iliacalaneurysma interna rechts } \\
\text { Z.n. Aneurysmaresektion mit Patchplastik Iliaca } \\
\text { communis rechts } \\
\text { Arterielle Hypertonie } \\
\text { Koronare 3-Gefäß-Erkrankung, Z.n. Stent }\end{array}$ & 26,7 & 8,5 & 284 & 44,3 \\
\hline 3 & $\begin{array}{l}67 \\
M\end{array}$ & $\begin{array}{l}\text { Wiederholtes Beatmungs- } \\
\text { leck, Beatmung in Bauchla- } \\
\text { ge eingeschränkt, ECMO }\end{array}$ & $\begin{array}{l}\text { Lungenarterienembolie bei tiefer Beinvenen- } \\
\text { thrombose links } \\
\text { akutes Nierenversagen, } \\
\text { Sulcale Blutauflagerungen rechts frontal und } \\
\text { parietal }\end{array}$ & 25,7 & 9,2 & 42 & 50 \\
\hline 4 & $\begin{array}{l}77 \\
M\end{array}$ & $\begin{array}{l}\text { Prolongierte ET-Intubation } \\
\text { Frustranes Weaning }\end{array}$ & $\begin{array}{l}\text { Koronare Herzerkrankung } \\
\text { Z.n. PTCA und DE-Stenting } \\
\text { Diabetes mellitus Typ II } \\
\text { Spinalkanalstenose Z.n. Dekompressionsopera- } \\
\text { tion } \\
\text { Z.n. Hüfttotalendoprothese }\end{array}$ & 29,1 & 7,7 & 249 & 53 \\
\hline 5 & $\begin{array}{l}76 \\
M\end{array}$ & $\begin{array}{l}\text { Prolongierte ET-Intubation } \\
\text { Frustranes Weaning }\end{array}$ & $\begin{array}{l}\text { Peripherer arterieller Verschlusskrankheit Stadi- } \\
\text { um IV nach Fontaine, } \\
\text { Z. n. popliteocruralem Venenbypass } \\
\text { Paroxysmales VHF } \\
\text { Schrittmacher bei Sick-Sinus-Syndrom } \\
\text { Arterielle Hypertonie }\end{array}$ & 19,4 & 9,1 & 302 & 56,5 \\
\hline
\end{tabular}

Rachen-Abstrichuntersuchung (SARSCOV-2 PCR) der beteiligten Mitarbeiter. Im Rahmen eines Mitarbeiterscreenings wurden alle $\mathrm{HNO}$-Operateure präoperativ im Nasen-Rachen-Abstrich aufSARSCoV-2 sowie SARS-CoV-2-IgG (ELISA) negativ getestet. Nach aktuellem Kenntnisstand haben sich infolge der TS bei dem medizinischen Personal 3 Wochen postoperativ weder Symptome gezeigt noch wurde im Fall einer Testung eine COVID-19-Infektion nachgewiesen.

Die Deutsche Gesellschaft für Anästhesiologie und Intensivmedizin (DGAI) empfiehlt in ihren S3 (2017) bzw. S2kLeitlinien (2019) eine TS für Patienten mit der dauerhaften Notwendigkeit einer invasiven Beatmung und fehlenden Optionen zur nichtinvasiven Ventilation (NIV) sowie bei allen anderen intubierten, invasiv beatmeten Patienten mit klinisch beobachteten prolongierten Weaning oder Beatmung länger als 7 Tage nach den ersten erfolglosen Spontanatmungsversuchen (,spontaneous breathing trial“, SBT) zur Reduktion der, mit der endotrachealen Intubation assoziierten Komplikationen (tracheale und laryngeale Schädigungen und erhöhtes Infektionsrisiko der unteren Atemwege; [1, 6, 26, 29]). Weitere Argumente für die TS sind die Verkürzung der Dauer einer nachteiligen Sedierung, die zur Toleranzsteigerung bei liegendem endotrachealen Tubus erforderlich ist, und damit eine Vermeidung der sonst erforderlichen „On-/off-Situation“ durch Intubation/Extubation mit einer zusätzlich möglichen Erhöhung der Sterblichkeit nach einem Extubationsversagen mit erforderlicher Reintubation [31]. Zusätzlich werden auch SBT erleichtert und damit die Beatmungszeit verkürzt. Hierbei gilt es, den Zeitpunkt der TS individuell zu wählen [2]. In Abhängigkeit von der Latenz zur Intubation werden frühe ( $<7$ Tage) von späten (ab 8-10 Tagen) TS unterschieden [29]. Frühe TS werden in den Leitlinien mit hoher Evidenz nicht empfohlen [1]. Ausgenommen davon sind TS aufgrund dauerhafter Notwendigkeit einer TS.
In der zweiten Version der S1-Leitline „Empfehlungen der intensivmedizinischen Therapie von Patienten mit Covid 19" der DGAI wird die Indikation und Methode zur Durchführung einer TS im Sinne einer Einzelfallentscheidung empfohlen und berücksichtigt den speziellen Umgang des Weanings bei COVID-19Patienten nicht [34].

International wird eine prolongierte invasive mechanische Beatmung bei COVID-19-Patienten, von bis zu 21 Tagen und länger propagiert, mit dem Ziel, möglichst einen mehrfach negativen SARS-CoV-2-Abstrich zu erreichen $[12,14,38]$. Ein Virusnachweis aus dem Respirationstrakt war bei ITS-Patienten sogar bis 31 Tage nach Symptombeginn möglich [9]. Grundsätzlich müssen aus unserer Erfahrung derzeit bei jeder TS im Verlauf einer COVID-19-Erkrankung trotz einmalig negativen SARS-CoV-2Nachweises noch eine potenzielle Viruslast angenommen und deshalb die erforderlichen hygienischen Erfordernisse beachtet werden. 


\section{Originalien}

Symptom-beginn

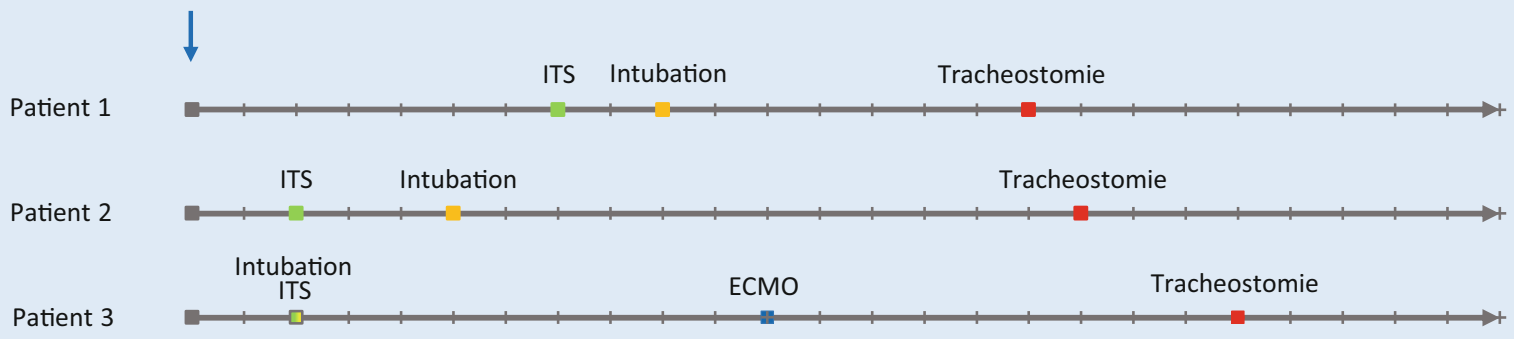

Patient $4 \quad$ ITS Intubation

$\begin{array}{lll}\text { ITS Intubation } & \text { Tracheostomie }\end{array}$

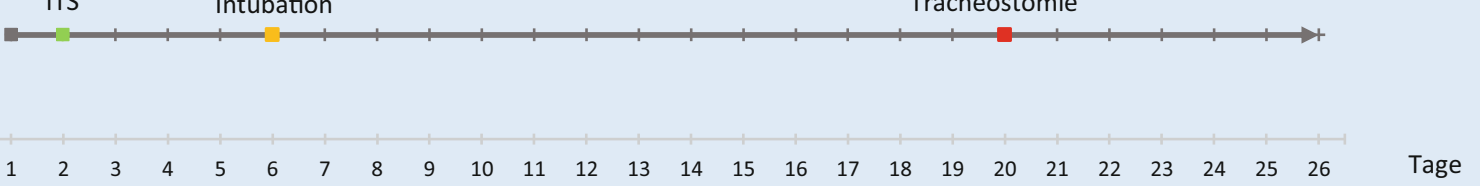

Abb. 3 \ Zeitlicher Verlauf des Atemwegmanagements von 5 COVID-19-Erkrankten. (ITS Intensivstation, ECMO extrakorporale Membranoxygenierung)

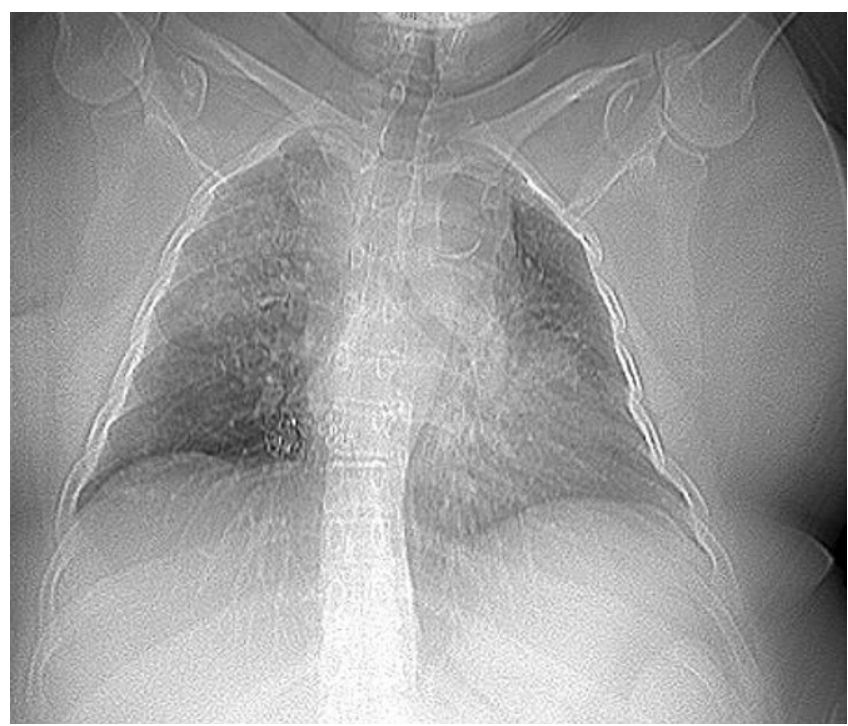

Abb. 4 \ Röntgen anterior-posterior im Liegen, multiple beidseitige Infiltrate bei COVID-19-Erkrankten (Patient 1)

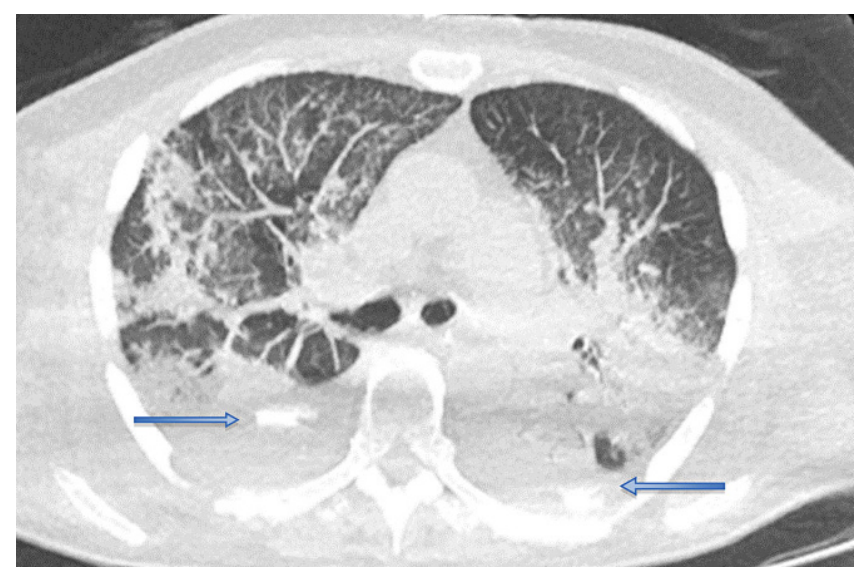

Abb. $5 \Delta$ CT-Thorax axial nativ bei COVID-19-Erkranktem (Patient 3), Konsolidierungen in den Unterlappen beidseits mit milchglasartigen Infiltraten ("crazy paving pattern"), Pleuraergüssen bds., einliegenden Thoraxdrainagen (Pfeile)
Aufgrund fehlender Prognoseparameter für die Beatmungsdauer von COVID-19-Patienten kann es momentan keine Empfehlung für den optimalen Zeitpunkt einer TS geben. Zudem stehen erfolgversprechende medikamentöse Therapien der Erkrankung und die abschließende Bewertung von Antikörperuntersuchungen aus. Somit wird die Anlage eines plastischen TS bis auf weiteres eine interdisziplinäre Einzelfall- entscheidung bleiben [21]. In manchen Fällen sind aufgrund der erschwerten respiratorischen Situation mit permanentem Lagewechsel bereits zu einem frühen, d.h. sehr aktiven Zeitpunkt der Erkrankung eine TS erforderlich. Auch die Beatmung in Bauchlage ist mit TK möglich. Die Vorteile der TS bei Langzeitbeatmung in der prolongierten Weaningphase durch Verkürzung der täglichen Aufwachphasen für SBT, ver- minderten Sedierungsbedarf ohne ET, Reduktion des Totraumvolumens, des Atemwegwiderstandes und der damit verbundenen Atemarbeit [29] überwiegen auch für COVID-19-Patienten. Bei hohem Patientenaufkommen können dadurch möglicherweise zeitnaher wieder freie Respiratorplätze für neue Patienten zur Verfügung stehen. Zum Schutz des Personals vor nosokomialen Infektionen sollte der Zeitraum einer 
geringeren COVID-19-Erkrankungsaktivität bei der Wahl des Zeitpunktes der TS situationsangepasst Berücksichtigung finden.

Die Durchführung einer TS bei COVID-19-Erkrankten im interdisziplinären Kontext und mit Unterstützung der Abläufe durch eine klinikinterne SOP und deren Training, ermöglicht einen sicheren Ablauf für Patient und das medizinisches Personal. Insbesondere eine SOP-gestützte unmissverständliche Kommunikation ( Abb. 2) kann eine akzidentielle unkontrollierte Aerosolbildung, wie bei Botti et al. beschrieben, verhindern [7].

Auch bei Beachtung aller Schutzmaßnahmen sind Restrisiken für das Personal jedoch nie vollständig ausgeschlossen. Deshalb sind eine engmaschige Symptomselbstkontrolle und Testungen des Personals auf SARS-COV-2 im Nasen-Rachen-Abstrich bei Auftreten von Symptomen empfehlenswert, um frühzeitig Infektionen zu detektieren.

Aus eigener Erfahrung sehen wir klinikintern Vorteile für die Durchführung einer TS im Operationssaal, da er optimale räumliche als auch interventionelle Voraussetzungen für diesen Eingriff unter besonderem Personalschutz bietet. Insbesondere bei hoher Auslastung der Intensivstationen führt eine Intervention am Patientenbett zu zusätzlicher ITS-Personalbindung bei beschränkter räumlicher Kapazität (ECMO, Dialyseeinheit) sowohl für das Operationsteam als auch das Operationsequipment (steriler Operationstisch, Sauger, bipolare Koagulation, Lichtquelle). Für eine TS von COVID19-Patienten muss sowohl im Operationssaal als auch auf der ITS insbesondere aufgrund der Schutzmaßnahmen ein gesteigerter Zeitaufwand eingeplant werden.

Grundsätzlich sollten Notfalltracheostomien mit unbekanntem COVID-Status mit allen hier genannten vorsorgenden Schutzmaßnahmen erfolgen. Bei elektiven Tracheostomien wird zusätzlich eine präoperative PCR-Testung auf SARSCOV-2 und anschließende stationäre Quarantäne mit Mund-Nasen-Schutz bis zum Eingriff empfohlen.

\section{Fazit für die Praxis}

- Die Indikationsstellung und der Zeitpunkt einer Tracheostomie (TS) bei COVID-19-Patienten sind immer interdisziplinäre Entscheidungen.

- Logistische und personelle Vorbereitungen vor einer TS von COVID19-Patienten sind essenziell zur Vermeidung von Infektionen des medizinischen Personals insbesondere in Notfallsituationen.

- Unabdingbar ist eine permanente Kommunikation des Operationsteams über den Operationsfortschritt sowie anästhesiologischer bzw. intraoperativer Probleme.

- Klinikinterne interdisziplinäre SOPs (Standard Operation Procedure) ermöglichen ein strukturelles Vorgehen, eine Feedbackkontrolle und nach konsequenter Prüfung eine Adaptation derselben.

\section{Korrespondenzadresse}

\section{Dr. A. Pudszuhn}

Klinik für Hals-, Nasen-, Ohrenheilkunde, Campus Benjamin Franklin, Charité Universitätsmedizin Berlin Hindenburgdamm 30, 12203 Berlin, Deutschland

annett.pudszuhn@charite.de

Funding. Open Access funding provided by Projekt DEAL.

\section{Einhaltung ethischer Richtlinien}

Interessenkonflikt. A. Pudszuhn, S. Voegeler, C. Berger, S. Treskatsch, S. Angermair, S. Hansen und V.M. Hofmann geben an, dass kein Interessenkonflikt besteht.

Für diesen Beitrag wurden von den Autoren keine Studien an Menschen oder Tieren durchgeführt. Für die aufgeführten Studien gelten die jeweils dort angegebenen ethischen Richtlinien.

Open Access Dieser Artikel wird unter der Creative Commons Namensnennung 4.0 International Lizenz veröffentlicht, welche die Nutzung, Vervielfältigung, Bearbeitung, Verbreitung und Wiedergabe in jeglichem Medium und Format erlaubt, sofern Sie den/die ursprünglichen Autor(en) und die Quelle ordnungsgemäß nennen, einen Link zur Creative Commons Lizenz beifügen und angeben, ob Änderungen vorgenommen wurden.

Die in diesem Artikel enthaltenen Bilder und sonstiges Drittmaterial unterliegen ebenfalls der genannten Creative Commons Lizenz, sofern sich aus der Abbildungslegende nichts anderes ergibt. Sofern das be- treffende Material nicht unter der genannten Creative Commons Lizenz steht und die betreffende Handlung nicht nach gesetzlichen Vorschriften erlaubt ist, ist für die oben aufgeführten Weiterverwendungen des Materials die Einwilligung des jeweiligen Rechteinhabers einzuholen.

Weitere Details zur Lizenz entnehmen Sie bitte der Lizenzinformation auf http://creativecommons.org/ licenses/by/4.0/deed.de.

\section{Literatur}

1. Deutsche Gesellschaft für Anästhesiologie und Intensivmedizin (DGAI), S3-Leitlinie Invasive Beatmung und Einsatz extrakorporaler Verfahren bei akuter respiratorischer Insuffizienz. 1. Auflage, Kurzversion, (Stand 5. Dez. 2017). https://www. awmf.org/uploads/tx_szleitlinien/001021k_S3_ Invasive_Beatmung_2017-12.pdf

2. World Health Organization (2020) Infection prevention and control during health care when novel coronavirus ( $\mathrm{nCoV}$ ) infection is suspected. https://www.who.int/publicationsdetail/infection-prevention-and-control-duringhealth-care-when-novel-coronavirus-(ncov)infection-is-suspected-20200125. Zugegriffen: 06.05.2020

3. World Health Organization (2020) (COVID-19). Situation Report-133. https://www.who.int/ docs/default-source/coronaviruse/situationreports/20200601-covid-19-sitrep-133.pdf? sfvrsn=9a56f2ac 4.Zugegriffen:01. Juni 2020

4. Balakrishnan K, Schechtman S, Hogikyan ND, Teoh AYB, McGrath B, Brenner MJ (2020) COVID19 pandemic: what every otolaryngologist-head and neck surgeon needs to know for safe airway management. Otolaryngol Head Neck Surg. https://doi.org/10.1177/0194599820919751

5. Bellizzi S, Panu Napodano CM, Salaris P, Pichierri G, Sotgiu G (2020) Regional variation in trajectories of health worker infections during the Covid-19 pandemic in Italy. Infect Control Hosp Epidemiol. https://doi.org/10.1017/ice.2020.189

6. Bishop MJ, Hibbard AJ, Fink BR, Vogel AM, Weymuller EA Jr. (1985) Laryngeal injury in a dog model of prolonged endotracheal intubation. Anesthesiology 62:770-773

7. Botti C, Lusetti F, Castellucci A, Costantini M, Ghidini A (2020) Safe tracheotomy for patients with COVID-19. Am J Otolaryngol. https://doi.org/ 10.1016/j.amjoto.2020.102533

8. Chee VW, Khoo ML, Lee SF, Lai YC, Chin NM (2004) Infection control measures for operative procedures in severe acute respiratory syndromerelated patients. Anesthesiology 100:1394-1398

9. Chen J, Qi T, Liu L, Ling Y, Qian Z, Li T, Li F, Xu Q, Zhang Y, Xu S, Song Z, Zeng Y, Shen Y, Shi Y, Zhu T, Lu H (2020) Clinical progression of patients with COVID-19 in Shanghai, China. J Infect 80:e1-e6

10. Coccolini F, Perrone G, Chiarugi M, Di Marzo F, Ansaloni L, Scandroglio I, Marini P, Zago M, De Paolis P, Forfori F, Agresta F, Puzziello A, D'Ugo D, Bignami E, Bellini V, Vitali P, Petrini F, Pifferi B, Corradi F, Tarasconi A, Pattonieri V, Bonati E, Tritapepe L, Agnoletti V, Corbella D, Sartelli M, Catena F (2020) Surgery in COVID-19 patients: operational directives. World J Emerg Surg 15:25

11. European Centre for Disease Prevention and Control (2020) Factsheet for health professionals on Coronaviruses. https://www.ecdc.europa.eu/en/ factsheet-health-professionals-coronaviruses. Zugegriffen: 05.05.2020 
12. David AP, Russell MD, El-Sayed IH, Russell MS(2020) Tracheostomy guidelines developed at a large academic medical center during the COVID-19 pandemic. Head Neck. https://doi.org/10.1002/ hed. 26191

13. Fakhry N, Schultz P, Moriniere S, Breuskin I, Bozec A, Vergez $S$, de Garbory L, Hartl D, Temam S, Lescanne E, Couloigner V, Barry B, French Society of Otorhinolaryngology $\mathrm{H}$, Neck S, French Society of H, Neck C (2020) French consensus on management of head and neck cancer surgery during COVID-19 pandemic. Eur Ann Otorhinolaryngol Head Neck Dis. https://doi. org/10.1016/j.anorl.2020.04.008

14. Faris C, Deben K, van Haesendonck G et al (2020) Making sense of personal protective equipment PPE and tracheostomy in the mists of the 2020 COVD-19 crisis. B-ENT. https://doi.org/10.5152/BENT.2020.20128

15. Geneva: World Helath Organization (2016.) Guidelines on core components of infection prevention and control programmes at the national and acute health care facility level. Licence CCBY-NC-SA 30 IGO

16. Givi B, Schiff BA, Chinn SB, Clayburgh D, lyer NG, Jalisi S, Moore MG, Nathan CA, Orloff LA, O’Neill JP, Parker N, Zender C, Morris LGT, Davies L (2020) Safety recommendations for evaluation and surgery of the head and neck during the COVID19 pandemic. JAMA Otolaryngol Head Neck Surg. https://doi.org/10.1001/jamaoto.2020.0780

17. Heyd CP, Desiato VM, Nguyen SA, O'Rourke AK Clemmens CS, Awad MI, Worley ML, Day TA (2020) Tracheostomy protocols during COVID-19 pandemic. Head Neck. https://doi.org/10.1002/ hed.26192

18. JacobT, Walker A, Mantelakis A, Gibbins N, Keane $O$ (2020) A framework for open tracheostomy in COVID-19 patients. Clin Otolaryngol. https://doi. org/10.1111/coa.13549

19. Klok FA, Kruip M, van der Meer NJM, Arbous MS, Gommers D, Kant KM, Kaptein FHJ, van Paassen J, Stals MAM, Huisman MV, Endeman H (2020) Confirmation of the high cumulative incidence of thrombotic complications in critically ill ICU patients with COVID-19: an updated analysis. Thromb Res. https://doi.org/10.1016/j.thromres. 2020.04.04

20. Kommission Ed, und $\mathrm{fK}$, Robert IKb, KochInstitut, fK, Robert IKb, Koch-Institut (2018) Prävention postoperativer Wundinfektionen. Bundesgesundheitsblatt 61:448-473

21. Lindemann J, Böhm F, Hoffmann T, Müller $A$, Jäckel $M$, Löwenheim $H$, Guntinas-Lichius $O$, Deitmer T, Dietz A (2020) Chirurgische Aspekte zur Tracheostomie bei Covid-19 positiven Patienten - Stellungnahme im Auftrag der Deutschen Gesellschaft für HNO-Heilkunde, Kopf- und Halschirurgie (DGHNO-KHC) und der AG Laryngologie \& Trachealerkrankungen. Laryngorhinootologie. https://doi.org/10.1055/a-1151-7932

22. Liu S, Chan TC, Chu YT, Wu JT, Geng X, Zhao N, Cheng W, Chen E, King CC (2016) Comparative epidemiology of human infections with middle east respiratory syndrome and severe acute respiratory syndrome Coronaviruses among Healthcare personnel. PLoSONE 11:e149988

23. Low JG, Wilder-Smith A (2005) Infectious respiratoryillnesses and their impact on healthcare workers: a review. Ann Acad Med Singapore 34:105-110

24. Portugal LG, Adams DR, Baroody FM, Agrawal N (2020) A surgical safety checklist for performing tracheotomy in patients with Coronavirus disease
19. Otolaryngol Head Neck Surg. https://doi.org/ $10.1177 / 0194599820922981$

25. Rabenau HF, Cinatl J, Morgenstern B, Bauer G, Preiser W, Doerr HW (2005) Stability and inactivation of SARS coronavirus. Med Microbiol Immunol 194:1-6

26. Rello J, Sonora R, Jubert P, Artigas A, Rue M, Valles (1996) Pneumonia in intubated patients: role of respiratory airway care. Am J Respir Crit Care Med 154:111-115

27. Robert Koch Institut (2020) Täglicher Lagebricht des RKI zur Corona-Virus-Krankheit-2019(COVID19). https://www.rki.de/DE/Content/InfAZ/ N/Neuartiges_Coronavirus/Situationsberichte/ 2020-05-05-de.pdf?_blob=publicationFile. Zugegriffen: 15.05.2020

28. Schälte GKF, Didion N, Pirlich N, Bickenbach J, Rehm M, Rossaint R (2020) Besonderheiten des Atemwegsmanagements bei Patienten mit vermuteter oder gesicherterCOVID-19Erkrankung und bei Patienten ohne Infektion während der Corona-Pandemie.Empfehlungen von DGAI und BDA. Anasth Intensivmed 61:S132-S136

29. Schönhofer BGJ, Braune S, Dellweg D, Fuchs H, Hirschfeld-Araujo J, Janssens U, Mörer O, Rollnik J, Rosseau S, Schreiter D, Weber-Carstens S, Windisch W, Westhoff M (2019) S2k Leitlinie Prolongiertes Weaning, AWMF-Register Nr. 020/015 https://www.awmf.org/uploads/tx_szleitlinien/ 020-015I_S2k_Prolongiertes_Weaning_2019_ 09_1.pdf.Zugegriffen: 26.05.2020

30. Schultz $P$, Morvan JB, Fakhry N, Moriniere $S$, Vergez S, Lacroix C, Bartier S, Barry B, Babin E, Couloigner V, Atallah I, French Society of Otorhinolaryngology HNS, French Society of Head NC (2020) French consensus regarding precautions during tracheostomy and post-tracheostomy care in the context of COVID-19 pandemic. Eur Ann Otorhinolaryngol Head Neck Dis. https://doi.org/ 10.1016/j.anorl.2020.04.006

31. Seymour CW, Martinez A, Christie JD, Fuchs BD (2004) The outcome of extubation failure in a community hospital intensive care unit: a cohort study. Crit Care 8:R322-R327

32. Skoog $\mathrm{H}$, Withrow $\mathrm{K}$, Jeyarajan $\mathrm{H}$, Greene B, Batra $\mathrm{H}$, Cox D, Pierce A, Grayson JW, Carroll WR (2020) Tracheotomy in the SARS-CoV-2 pandemic. Head Neck. https://doi.org/10.1002/hed.26214

33. Sommer DD, Engels PT, Weitzel EK, Khalili S, Corsten M, Tewfik MA, Fung K, Cote D, Gupta M, Sne N, Brown TFE, Paul J, Kost KM, Witterick IJ (2020) Recommendations from the CSO-HNS taskforce on performance of tracheotomy during the COVID-19 pandemic. J Otolaryngol Head Neck Surg 49:23

34. Kluge St, Janssens $U$, Welte $T$, Weber-Carstens St, Schälte G, Salzberger B, Gastmeier P, Langer F, Wepler M, Westhoff M, Pfeifer M, Hoffmann F, Böttiger BW, Marx G, Karagiannidis Ch (2020) S1-Empfehlungen zur intensivmedizinischen Therapie von Patienten mit COVID-19, AWMFReg.-Nr. 113/001. (Version 2, Stand 19.06.2020). https://www.awmf.org/uploads/tx_szleitlinien/ 113-001I_S1_Intensivmedizinische-Therapievon-Patienten-mit-COVID-19_2020-06_1.pdf. Zugegriffen: 22.07.2020

35. Tay JK, Khoo ML, Loh WS (2020) Surgical considerations for tracheostomy during the COVID-19 pandemic: lessons learned from the severeacute respiratory syndrome outbreak.JAMA Otolaryngol Head Neck Surg. https://doi.org/10. 1001/jamaoto.2020.0764

36. Thomas-Rüddel DWJ, Dickmann $P$, Ouart D, Kortgen A, Janssens UBM (2020) "Coronavirus disease 2019“ (COVID-19): update für Anästhesisten und Intensivmediziner März 2020. Anaesthesist 69:225-235. https://doi.org/10.1007/s00101020-00758-x

37. Tien HC, Chughtai T, Jogeklar A, Cooper AB, Brenneman $F$ (2005) Elective and emergency surgery in patients with severe acute respiratory syndrome (SARS). Can J Surg 48:71-74

38. Tran K, Cimon K, Severn M, Pessoa-Silva CL, Conly J (2012) Aerosol generating procedures and risk of transmission of acute respiratory infections to healthcare workers: a systematic review. Plos One 7:e35797

39. Ufficio Stampa e Informazione Federazione Nazionale degliOrdini dei MediciChirurghi e degliOdontoiatri 25 aprile AF (2020) II Coronavirus ci ha portato via i nostri anziani e i nostri medici, non estingua la nostra memoria. https://portale.fnomceo.it/ 25-aprile-anelli-fnomceo-il-coronavirus-ci-haportato-via-i-nostri-anziani-e-i-nostri-medicinon-estingua-la-nostra-memoria/. Zugegriffen: 01.05.2020

40. van Doremalen $\mathrm{N}$, Bushmaker $\mathrm{T}$, Morris $\mathrm{DH}$, Holbrook MG, Gamble A, Williamson BN, Tamin A, Harcourt JL, Thornburg NJ, Gerber SI, LloydSmith JO, de Wit E, Munster VJ (2020) Aerosol and surface stability of SARS-coV-2 as compared with SARS-coV-1.N Engl J Med 382:1564-1567 